\title{
Nasal, retronasal, and gustatory perception: An experimental comparison
}

\author{
KONRAD J. BURDACH \\ University of Munich, Munich, Federal Republic of Germany \\ and \\ JAN H. A. KROEZE and E. P. KÖSTER \\ Utrecht University, Utrecht, The Netherlands
}

\begin{abstract}
Four odor substances (lemon aroma, rum aroma, ethyl butyrate, and amyl acetate) in different concentrations were presented nasally and orally (retronasally) in a four-alternative forced-choice procedure (detection tasks). In a further experimental condition, sucrose was added to the stimuli. Finally, the taste properties of the (nonsugared) odor stimuli were judged on seven semantic scales. In the detection of odor-containing stimuli, there were no significant differences between nasal and retronasal stimulation. In the taste conditions, however, there was a significant decline of "hits" when the stimuli contained sucrose in addition to the odor substances. Furthermore, in semantic scaling of the taste stimuli, a new variant of the taste-smell illusion was observed, namely, a tendency to attribute "suited" basic taste categories (e.g., "sweet") to pure (nongustatory) odor stimuli.
\end{abstract}

Up to now, relations and interactions between taste and smell have not attracted much attention from chemosensory research. On the contrary: From the titles of the important chemoreception textbooks (e.g., Beidler, 1971; Carterette \& Friedman, 1978), one may even obtain the impression of completely independent senses. But, although the taste-smell division may seem convincing from an anatomical point of view, there are growing doubts about its ecological and behavioral validity. In an overwhelming number of real-life situations, especially in nutritional contexts, both senses are addressed simultaneously or in close sequential order. Thus, a rigid separation in two independent research streams may ignore essential relations between taste and smell which might turn out to be especially important for the practical application of research results.

Indeed, "pure" olfactory (nasal) processes undoubtedly have to be considered biologically relevant (e.g., in reproduction processes or in food search). However, although "pure" taste (nonolfactory) stimuli, such as sucrose or sodium chloride, are studied very frequently in laboratory taste experiments, they are seldom encountered in real-life nutritional contexts. Perhaps the ecological validity of taste research can be increased substantially by redefining research topics in closer agreement with the prescientific experience, taking into account that olfaction and gustation combined form the sense of taste-and this is what we tried to do in designing the present study.

The first author's mailing address is: Wastelbauerstr. 4, 8000 Munchen 60 , Federal Republic of Germany. The second and third authors' mailing address is: Psychological Laboratory, Utrecht University, Utrecht, The Netherlands.
What is known about the relations between nasal, retronasal, and gustatory perception is roughly the following: Although orally presented (retronasal) odor stimuli seem to evoke oral sensations, in fact the odorous component is analyzed mainly by receptors of the nasal cavity (Hyman, Metzer, \& Calderone, 1979; Murphy \& Cain, 1980), a taste-smell illusion occurs. At weak-tomoderate concentrations, the aroma (odor component) disappears-though not completely-if the nostrils are closed (Murphy, Cain, \& Bartoshuk, 1977) or if the participation of the nasal sensory system is prevented in some other way (Mozell, Smith, Smith, Sullivan, \& Swender, 1969). At high concentrations of aroma stimuli, such as vanillin or piperonal, however, the gustatory component becomes the salient feature (Cometto-Muñiz, 1981).

On the other hand, although the receptors of the nasal cavity seem to play a dominant role in aroma perception, there are some foods that do not smell very pleasant but have a very good taste, for example, Limburger cheese (Rozin, 1982). This experience may be related to a functional difference between nasal and retronasal perception of odorous stimuli.

In the literature, there is no evidence for an interaction between taste and odor perception (Hornung \& Melvin, 1984; Murphy \& Cain, 1980). The authors mentioned above came to the conclusion that the perceived intensity of taste and aroma combined-flavor-corresponds approximately to the sum of the isolated odor and taste components. Furthermore, Murphy and Cain (1980) found no effect of the "harmony" of the odor-taste mixture (e.g., citral plus sucrose vs. citral plus $\mathrm{NaCl}$ ). Garcia-Medina (1981) also concluded that "neither taste nor smell contributes to flavor at the expense of the other" (p. 13), but 
proposed a vector summation model for the determination of flavor magnitude. These results contrast with observations of interactions between the olfactory and the (nasal) trigeminal system: Cain and Murphy (1980) found significant mutual inhibition between smell and irritation.

The experiments to be reported here focus on two questions: (1) Are there any systematic differences in the detection of weak odor stimuli, presented either retronasally (orally) or prenasally? (2) Does a simultaneous (gustatory) taste stimulus influence retronasal olfactory sensitivity?

Two experiments with different subjects and slightly different experimental procedures were carried out. The second experiment (main experiment) may be seen as the methodologically improved replication of the first (prestudy).

\section{METHOD}

\section{Subjects}

Fifteen subjects, high school and university students ( 5 males and 10 females), participated in the prestudy. Their ages ranged from 16 to 35 years (mean: 21.2 years). Twenty-seven different subjects (14 males and 13 females) from the same population participated in the main experiment. They were between 16 and 33 years old (mean: 22.8 years). None of the experimental subjects had had any experience with chemosensory experiments.

\section{Stimuli}

Four substances were used: ethyl butyrate, rum aroma, ${ }^{1}$ lemon aroma, ${ }^{1}$ and amyl acetate, each in seven concentrations. In the prestudy, the highest concentrations were $5 \times 10^{-4} \mathrm{vol}$. \% for lemon, $5 \times 10^{-6} \mathrm{vol}$. \% for rum, and $10^{-7}$ vol. \% for ethyl butyrate and amyl acetate. Each next weaker concentration was 5 times weaker in the dilution series.

In the main experiment, the highest concentrations were $\sqrt{10} \times 10^{-6}$ vol. \% for lemon, $\sqrt{10} \times 10^{-7}$ vol. $\%$ for rum, and $\sqrt{10} \times 10^{-9}$ vol. $\%$ for ethyl butyrate and amyl acetate. Each step was $\sqrt{10}$ times weaker than the foregoing in the dilution series.

All solutions were prepared with tap water ${ }^{2}$ or sugared tap water $(0.128 \mathrm{M}$ sucrose in both experiments). Tap water was used as a solvent since, in our experiences and those of Rothe, Tunger, and Sieber (1972), tap water has seemed to be superior to distilled water in aroma research because of better stimulus constancy (e.g., lack of off-odors).

In the smell conditions (see next section), the stimuli were offered in 250-ml erlenmeyer bottles containing $200 \mathrm{ml}$ of stimulus substance (sniff-bottle technique). In the taste conditions (see next section), $450 \mathrm{ml}$ of test substance was offered in plastic cups. In these conditions, the subjects used a straw to draw the substance out of the cup. In order to prevent stimulation through the nostrils, the cups were sealed off by foil in which a little hole permitted the introduction of a straw.

The 28 different stimuli ( 4 substances $\times 7$ intensity levels) were offered in a four-alternative forced-choice paradigm (4AFC), which led to a total of 112 presentations per condition. The "blanks" contained the solvent. Intensity levels and substances were randomized with the restriction that the same substance not occur twice in immediate succession. Between each of the $284 \mathrm{AFC}$ trials, a recovery time of 30-40 sec was allowed. During the taste conditions, the subjects rinsed their mouths thoroughly with tap water after each trial.

\section{Experimental Conditions}

There were four experimental conditions: (1) smell, water-the subjects tried to detect the substances by smelling (the solvent was tap water); (2) smell, sugared water (the solvent was sugared tap water); (3) taste, water-the subjects tried to detect the substances by tasting (retronasal stimulation); and (4) taste, sugared water-identical to (3), except for the solvent, which was sugared tap water.

The prestudy contained only Conditions 1,3 , and 4 ; the main experiment contained all four conditions.
Before going through the detection tasks, the subjects of the prestudy tasted the 28 odor-containing stimuli (as well as two "blanks") and assessed them on seven different semantic scales-sweet, salty, bitter, sour, fruity, aromatic, and pleasant. The intensity of each attribute was expressed on a 5-point scale. These ratings were included to obtain information about the subjective quality of the test substances, especially about the relationship between gustatory and olfactory sensations.

\section{Sessions}

Both experiments were carried out in two sessions of approximately 90 min each. The second session took place 1 week after the first. Each session was divided in two subsessions with a break of $10 \mathrm{~min}$ in between.

In the first session of the prestudy, the subjects began with the semantic assessments. After the break, Condition 4 was carried out. The second session was started with Condition 1; after the break, Condition 3 was run. All subjects went through the conditions in the same order.

The main experiment contained only the four detection conditions. Now each subject got a random order of the four conditions. The main sessions were also split up into two subsessions, each subsession now containing one experimental condition per subject.

\section{RESULTS}

The results of the semantic assessments are summarized in Figure 1. Leaving aside the "noise" of the lower concentrations, we see that generally the aroma perception, represented by the categories "fruity" and "aromatic," tend to be more intense than the basic taste categories. Some taste sensations, however, were rated surprisingly high, if one takes into account that even the strongest concentrations were still so weak that we can practically exclude any equivalent stimulus property, for example, for the sweet component in amyl acetate. Apparently, we observe here a subjective attribution that is not based on a measurable gustatory stimulus.

Before calculating the experimental treatment effects, we examined whether the four test substances could be considered equivalent subtests of the subjects' ability to detect weak odor stimuli under the conditions described above. Comparison of the numbers of correct detections for the four substances (Table 1) showed that they were of about the same magnitudes within the experimental conditions. The scores turned out generally higher in the prestudy because of the higher stimulus concentrations. Furthermore, for each substance, the average intercorrelation with the three remaining substances (Spearman rank correlation) was computed separately for the taste and smell conditions of the main experiment. The substances show quite homogeneous intercorrelations (Table 2), which, however, turned out to be generally lower in the smell conditions.

The statistical test of the differences between experimental treatments was based on the number of detections per condition, pooled over substances and corrected for chance. The formula used to correct for chance was

$$
f_{\text {corr }}=28\left(f_{o}-f_{c}\right) /\left(28-f_{x}\right),
$$

in which $f_{o}$ is the observed frequency of hits and $f_{c}$ the number of detections expected on a chance level. The corrected values were rounded. The medians of the four ex- 

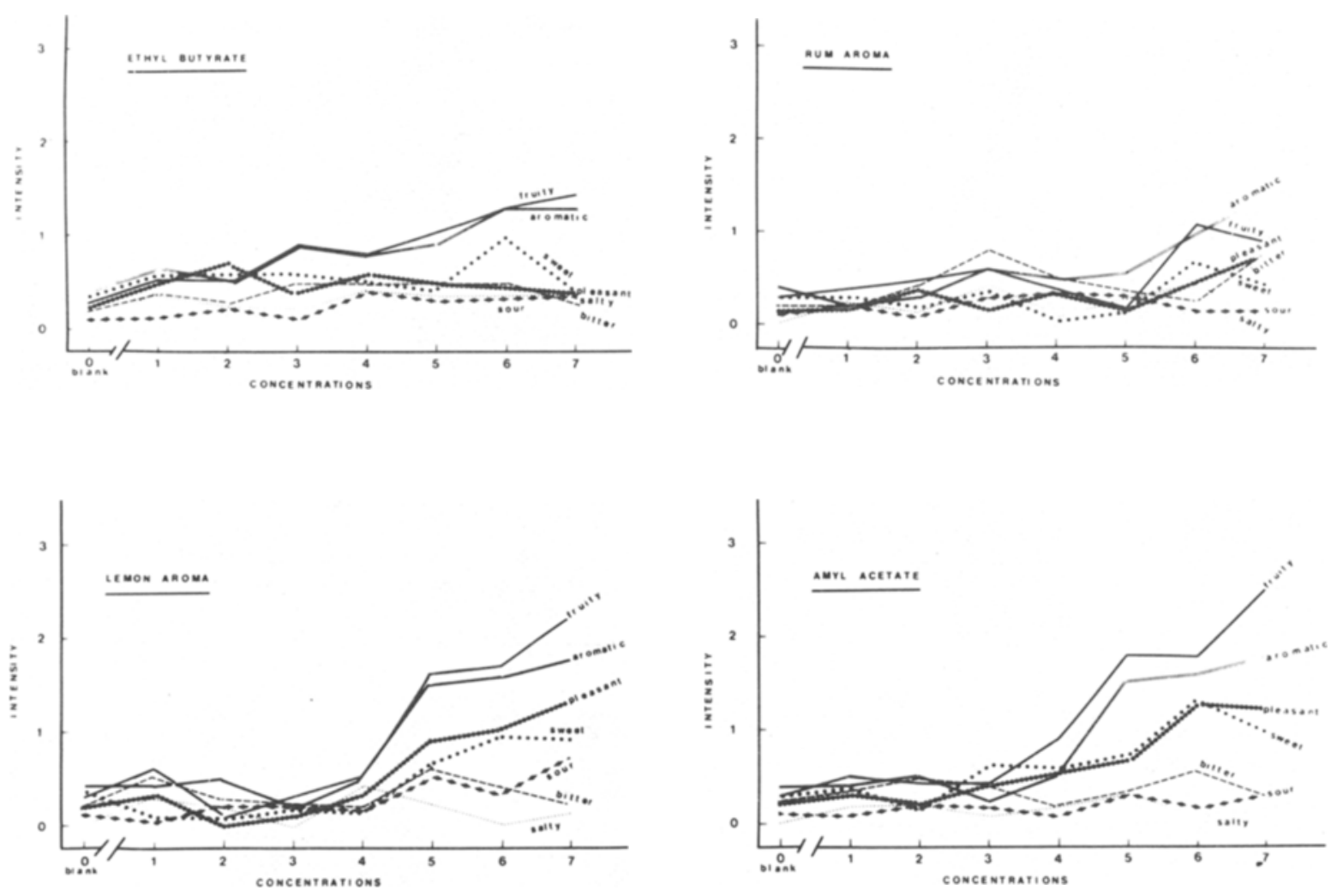

Figure 1. The semantic assessment of the four test substances, based on the arithmetical means of the taste judgments $(\mathrm{N}=15)$. Concentration 1 corresponds to the weakest concentration, concentration 7 to the strongest (see description of the stimuli). The intensity was measured on a 5 -point scale $(0=$ no sensation at all; $4=$ strong sensation of the type indicated by the attribute name).

perimental conditions turned out as follows (medians of the prestudy in brackets): smell, water-23 (27); smell, sugared water-25; taste, water-20 (27); and taste, sugared water-11 (20).

Table 1

Mean Detection Scores of the Test Substances

\begin{tabular}{lcccc}
\hline & \multicolumn{4}{c}{ Conditions } \\
\cline { 2 - 5 } \multicolumn{1}{c}{ Substance } & $\begin{array}{l}\text { Smell, } \\
\text { Water }\end{array}$ & $\begin{array}{c}\text { Smell, } \\
\text { Sugared Water }\end{array}$ & $\begin{array}{c}\text { Taste, } \\
\text { Water }\end{array}$ & $\begin{array}{c}\text { Taste, } \\
\text { Sugared Water }\end{array}$ \\
\hline \multicolumn{4}{c}{ Prestudy $(\mathrm{N}=15)$} \\
Ethyl Butyrate & 6.80 & & 6.70 & 5.44 \\
Rum & 6.41 & 6.03 & 4.50 \\
Lemon & 6.57 & 6.23 & 4.71 \\
Amyl Acetate & 6.42 & & 6.44 & 5.62 \\
& Main & & \\
Ethyl Butyrate & 6.38 & 5.93 & 5.23 & 4.69 \\
Rum & 5.30 & 5.91 & 5.53 & 4.23 \\
Lemon & 5.84 & 5.55 & 6.12 & 3.90 \\
Amyl Acetate & 5.74 & 5.54 & 4.60 & 4.25 \\
\hline
\end{tabular}

Table 2

Average Intercorrelations of the Test Substances

\begin{tabular}{ccccc}
\hline Condition & Ethyl Butyrate & Rum & Lemon & Amyl Acetate \\
\hline Smell & .52 & .63 & .57 & .68 \\
Taste & .89 & .86 & .89 & .79 \\
\hline
\end{tabular}

Note-For each substance, the average Spearman rank correlation with the remaining three substances is indicated. The two smell conditions and the two taste conditions are combined (data from main experiment).
A Friedman one-way analysis of variance (Siegel, 1956) revealed a significant effect of experimental treatments [main experiment, $\chi^{2}(3)=16.01, p<.01$; prestudy, $\chi^{2}(2)$ $=12.23, \mathrm{p}<.01]$. The results of a further analysis of the treatments in pairs by Wilcoxon tests (Siegel, 1956) are shown in Table 3. It may be concluded from these tests that the significance of the Friedman analysis is caused by the low performance level in the taste/sugared-water condition, which, in both experiments differs significantly from each of the other conditions.

\section{DISCUSSION}

Is there a functional equivalence between retronasal and nasal odor perception? In neither experiment did we find significant differences between the smell-water and the taste-water condition; so the results seem to be in favor of this equivalence. One should not forget, however, that this study focused on quantitative aspects: the sensitivity of nasal and retronasal sensory functions was measured. We still do not have any information about qualitative aspects, for example, qualitative differences between nasal and retronasal sensations. All we can say at the moment is that, irrespective of the locus of stimulation, nasal and oral odor stimuli evoke (quantitatively) similar sensations, probably because both sensory functions are based on the same neurophysiological units, namely the olfactory and (nasal) trigeminal systems (Ist and Vth cranial nerves). 
Table 3

Comparison of the Experimental Treatment Effects

\begin{tabular}{lcccc}
\hline \multicolumn{1}{c}{ Condition } & Smell, & Smell, & Taste, & Taste, \\
& Water Sugared Water & Water & Sugared Water \\
\hline Smell, Water & - & .673 & 1.634 & $3.315^{*}$ \\
& & & $(.866)$ & $\left(2.868^{*}\right)$ \\
Smell, Sugared Water & & - & 1.538 & $3.123^{*}$ \\
Taste, Water & & & - & $3.087^{*}$ \\
& & & & $\left(2.953^{*}\right)$ \\
Taste, Sugared Water & & & & -
\end{tabular}

Note-Each experimental condition was tested against the remaining three conditions by Wilcoxon's test (Wilcoxon's $Z$ values are indicated). The results of the prestudy are presented in parentheses. ${ }^{*} p<.01$.

The semantic scaling of aroma stimuli in some cases revealed a "subjective" interaction phenomenon, a tendency to add (apparently nonexistent) basic taste components that harmonize with the perceived aroma stimulus (e.g., "sweet" to the aroma of amyl acetate). That-apart from the illusion regarding the locus of stimulation (mouth instead of nose) - a pure aroma stimulus tends to elicit, in addition to the expected olfactory sensation, a gustatory response may be considered another aspect of the taste-smell illusion.

Finally, according to our results, there is a definite suppression of retronasal aroma perception by gustation: we observed significantly more errors in aroma detection tasks as soon as the olfactory and the gustatory systems were addressed simultaneously. These findings contrast with those of Garcia-Medina (1981), Hornung and Melvin (1984), and Murphy and Cain (1980), who found almost no interaction between olfaction and gustation. This discrepancy may be related to the different methodologies involved: the authors mentioned above used magnitude estimation procedures, whereas we measured the sensitivity of the experimental subjects by applying methods that correspond more closely to those of test psychology. Our approach may be rated as less general because only relatively weak aroma stimuli were combined with only one (moderately strong) gustatory stimulus, whereas the authors cited above covered many more pos- sible stimulus combinations. On the other hand, our results are based on fairly objective and reliable sensory tests, whereas their results rest on subjective scaling procedures that may produce less reliable findings. So, for the time being, this discrepancy remains unclear but may help to stimulate further research.

\section{REFERENCES}

BeidLer, L. M. (1971). Handbook of sensory physiology (Vol. 4. Part 1, Olfaction; Part 2, Taste). Berlin: Springer.

CAIN, W. S., \& MURPHY, C. L. (1980). Interaction between chemoreceptive modalities of odor and irritation. Nature, 284, 255-257.

Carterette, E. C., \& Friedman, M. P. (Eds.) (1978). Handbook of Perception (Vol. GA) Tasting and smelling. New York: Academic Press.

Cometto-Muniz, E. J. (1981). Odor, taste, and flavor perception of some flavoring agents. Chemical Senses, 6, 215-223.

Garcia-Medina, M. R. (1981). Flavor-odor taste interactions in solutions of acetic acid and coffee. Chemical Senses, 6, 13-22.

Hornung, D. E., \& MElvin, P. E. (1984). The independence and integration of olfaction and taste. Chemical Senses, 9, 97-106.

Hyman, A., Metzer, T., \& Calderone, L. (1979). The contribution of olfaction to taste discrimination. Bulletin of the Psychonomic Society, 13, 359-362.

Mozell, M. M., Smith, B. P., Smith, P. E., Sullivan, R. J., JR., \& SWENDER, P. (1969). Nasal chemoreception in flavor identification. Archives of Otolaryngology, 90, 131-137.

Murphy, C., \& CAIN, W. S. (1980). Taste and olfaction: Independence versus interaction. Physiology \& Behavior, 24, 601-605.

MurPhy, C., Cain, W. S., \& Bartoshuk, L. M. (1977). Mutual action of taste and olfaction. Sensory Processes, 1, 204-211.

Rothe, M., Tunger, L., \& SiEBer, H. J. (1972). Schwellenkonzentrationen von Aromastoffen und ihre Nutzung zur Auswertung von Aromaanalysen. Die Nahrung, 16, 483-495.

RozIN, P. (1982). "Taste-smell confusions" and the duality of the olfactory sense. Perception \& Psychophysics, 31, 397-401.

SIEGEL, S. (1956). Nonparametric statistics. New York: McGraw-Hill.

\section{NOTES}

1. Product of Ernst Müller \& Co., Neutraubling, West Germany.

2. Munich tap water of June 19 and 26, 1982 (prestudy) and of December 10 and 17, 1982 (main experiment).

(Manuscript received May 11, 1984; accepted for publication June 24, 1984.) 